\title{
Considerações sobre o modelo experimental de hipertensão intracraniana e avaliação do sistema de microchip para monitoração de pressão epidural
}

\author{
Wellingson Silva Paiva', Almir Ferreira de Andrade'2, Alessandro Rodrigo Belon ${ }^{3}$, \\ Edson Bor-Seng-Shu', Marcelo de Lima Oliveira ${ }^{3}$, Robson Luis Amorim ${ }^{4}$, \\ Matheus Schmidt Soares ${ }^{3,5}$, Jose Pinhata Otoch ${ }^{6}$, Manoel Jacobsen Teixeira ${ }^{7}$
}

Laboratório de Pesquisa em Cirurgia Experimental (LIM-26) da Faculdade de Medicina da Universidade de São Paulo (FMUSP), São Paulo, SP, Brasil e Divisão de Neurocirurgia do Hospital das Clínicas da FMUSP, São Paulo, SP, Brasil.

\section{RESUMO}

Objetivo: Neste trabalho temos o objetivo de avaliar a acurácia do sistema de aferição da pressão intracraniana (PIC) epidural com microchip. Métodos: Foram estudados 27 suínos sob anestesia geral, devidamente assistidos com monitoração ventilatória e hemodinâmica. Durante o experimento foi reproduzido um processo expansivo intracerebral programado no lobo frontal direito. O experimento constou de três grupos $(A, B$ e $C$ ) com hipertensão intracraniana gerada com balão reproduzindo um hematoma intracerebral. Em todos os grupos foram calibrados os parâmetros normais: os dois sistemas de PIC foram comparados e estudados quanto à correlação dos valores aferidos. Resultados: O comportamento médio da PIC ao longo dos momentos de avaliação foi estatisticamente diferente entre os grupos $(p<0,001)$. A reprodução de ressangramento resultou em elevação significativa da PIC $(p<0,001)$. Avaliando-se a acurácia comparativa geral, verificou-se um coeficiente de correlação intraclasse de 0,8. Conclusão: O modelo de hipertensão intracraniana por balão em suínos é factível e confiável na geração de hipertensão intracraniana. O sistema de aferição de pressão intracraniana epidural apresenta elevado coeficiente de correlação com o sistema de aferição parenquimatoso na avaliação global.

\section{PALAVRAS-CHAVE}

Hipertensão intracraniana, pressão intracraniana, espaço epidural, modelos animais, hemorragia cerebral.

\section{ABSTRACT}

Experimental model of intracranial hypertension and evaluation of the microchip system for epidural pressure monitoring of epidural pressure

Objective: In this paper we aim to evaluate the accuracy of the measurement with microchip epidural system. Methods: Twenty-seven pigs with were studied, under general anesthesia, properly assisted with ventilation and hemodynamic monitoring. During the experiment, we have simulated frontal intracerebral expansive process. The experiment consisted of three groups $(A, B$ and $C)$ with intracranial hypertension generated with the simulation of an intracerebral hematoma. The two systems were compared and studied as the correlation of the measured values. Results: The average behavior of the increased intracranial pressure (ICP) over the time points are statistically different between groups $(p<0.001)$. The simulation of rebleeding resulted in a significant increase in ICP $(p<0.001)$. Evaluating the overall comparative accuracy there was an intraclass correlation coefficient of 0.8 . Conclusion: The model of intracranial hypertension balloon in pigs is feasible and reliable in generating intracranial hypertension. The system for measuring intracranial epidural pressure has a high correlation coefficient with the system parenchymal gauging the overall evaluation.

\section{KEYWORDS}

Intracranial hypertension, intracranial pressure, epidural space, models animal, cerebral hemorrhage.

1 Médico supervisor da Divisão de Neurocirurgia do Hospital das Clínicas da Faculdade de Medicina da Universidade de São Paulo (HCFMUSP), São Paulo, SP, Brasil.

2 Diretor técnico da Unidade de Emergência da Neurocirurgia do HCFMUSP, São Paulo, SP, Brasil.

3 Pesquisador do Laboratório de Pesquisa em Cirurgia Experimental da FMUSP, São Paulo, SP, Brasil.

4 Médico-assistente da Divisão de Neurocirurgia do HCFMUSP, São Paulo, SP, Brasil.

5 Neurocirurgião da Santa Casa de Passos, Passos, MG, Brasil.

6 Professor-associado de Cirurgia da FMUSP, São Paulo, SP, Brasil.

7 Professor titular de Neurocirurgia da FMUSP, São Paulo, SP, Brasil. 


\section{Introdução}

O diagnóstico correto e precoce da hipertensão intracraniana (HIC) é essencial para a boa condução de medidas de neuroproteção, desde o estabelecimento da lesão cerebral primária até o seguimento pós-operatório em uma unidade de terapia intensiva. Para tanto, diversos dispositivos foram desenvolvidos com esse intuito. ${ }^{1}$ A monitoração invasiva da pressão intracraniana (PIC) é o único método aceito, indiscriminadamente, como forma para o diagnóstico seguro do aumento da pressão intracraniana, assim como para guiar o tratamento da HIC em algumas situações clínicas. Assim, a elaboração de um modelo in vivo de avaliação de hipertensão intracraniana e seus efeitos é essencial para entender a fisiopatologia da fase aguda da lesão cerebral e das medidas terapêuticas utilizadas., ${ }^{1,2}$

A medida da pressão ventricular ou parenquimatosa é o padrão de referência para a monitorização da PIC, contudo, as complicações infecciosas e hemorrágicas relacionadas ao uso de sistemas invasivos como o sistema ventricular e parenquimatoso fizeram surgir, historicamente, uma busca por um método menos invasivo e que produzisse dados confiáveis. ${ }^{2,3}$ Os métodos de monitoração epidural mais antigos não demonstraram resultados confiáveis. Entretanto, com o surgimento do sistema de aferição por microchip, ressuscitou-se a ideia do uso clínico desse método. Apesar da nova tecnologia, ainda há controvérsia, pois existem publicações indicando resultados inconstantes quanto à acurácia para uso clínico. Assim, existe uma necessidade de avaliação de acurácia que permita um uso clínico com adequada confiabilidade do método. Neste estudo temos o objetivo de avaliar o método epidural Neurodur ${ }^{\circledR}$ de aferição de pressão intracraniana comparado com o sistema padrão parenquimatoso de medida de pressão intracraniana.

\section{Material e métodos}

Foram estudados prospectivamente 27 suínos híbridos das raças Landrace, Duroc e Pietrain, com aproximadamente $20 \mathrm{~kg}$, sob anestesia geraI, devidamente assistidos com monitoração ventilatória e hemodinâmica. Este estudo foi aprovado pela Comissão de Ética em Pesquisa Animal.

\section{Preparação do animal: anestesia e monitoração}

Os suínos foram submetidos a 12 horas de jejum com livre acesso à água, até uma hora antes do experimento.
A técnica anestésica empregada em todos os animais consistia em administrar Quetamina (Ketamin- ${ }^{\circledR}$, Cristália) na dose de $5 \mathrm{mg} / \mathrm{kg}$ e Midazolam (Dormire ${ }^{\circledR}$, Cristália) na dose de $0,25 \mathrm{mg} / \mathrm{kg}$, ambos colocados em mesma seringa e administrados pela via intramuscular, como medicação pré-anestésica. Decorridos quinze minutos, a veia marginal da orelha foi cateterizada com cateter vascular de calibre 20 ou 22 (BD Insyte ${ }^{\mathrm{TM}}$ ). Uma vez estabelecido o acesso venoso, realizou-se a indução anestésica, que consistiu da aplicação de propofol (Provine ${ }^{\circledR} 1 \%$ - Cláris) na dose de $5 \mathrm{mg} / \mathrm{kg}$, e utilizou-se também um volume inicial de $20 \mathrm{ml} / \mathrm{kg}$ de solução fisiológica ( $\mathrm{NaCl}$ 0,9\%) para compensar a perda volêmica relativa ao jejum. Os animais foram entubados com sonda endotraqueal de diâmetro 6 (Portex $\left.{ }^{\circledR}\right)$, sendo a manutenção anestésica feita com propofol (Provine ${ }^{\circledR}$ $1 \%$ - Cláris) na dose de $3 \mathrm{mg} / \mathrm{kg} / \mathrm{h}$ e a analgesia mantida com fentanil (Fentanest ${ }^{\circledR}$ - Cristália), por uma dose inicial de $5 \mu \mathrm{g} / \mathrm{kg}$ seguida de infusão contínua de 0,4 $\mu \mathrm{g} / \mathrm{kg} / \mathrm{min}$ por via intravenosa (IV). O bloqueio neuromuscular foi obtido com a administração de pancurônio (Pancuron $^{\circledR}$, Cristália), em bólus na dose de $0,1 \mathrm{mg} / \mathrm{kg}$ IV seguida de infusão contínua desse agente na dose de $0,02 \mathrm{mg} / \mathrm{kg} / \mathrm{h}$.

Após entubação endotraqueal, os animais foram submetidos à ventilação mecânica controlada ciclada a volume (Ventilador Dixtal ${ }^{\circledR} 5010$ ), com volume corrente (VC) de $10 \mathrm{ml} / \mathrm{kg}$, fração inspirada de oxigênio $\left(\mathrm{FIO}_{2}\right)$ de 0,50 e pressão positiva expiratória final (PEEP) de $5 \mathrm{cmH}_{2} \mathrm{O}$. Os parâmetros ventilatórios foram ajustados para manter a $\mathrm{PaCO}_{2}$ entre $35 \mathrm{mmHg}$ e 45 $\mathrm{mmHg}$, a $\mathrm{PaO}_{2}$ entre $100 \mathrm{mmHG}$ e $150 \mathrm{mmHg}$ e o $\mathrm{pH}$ entre 7,35 e 7,45 .

\section{Procedimento experimental}

Durante o experimento foi simulado um processo expansivo intracerebral implantado no hemicrânio direito. Uma incisão frontotemporal foi realizada na cabeça do animal. Em seguida, efetuou-se a trepanação óssea $1 \mathrm{~cm}$ lateral à sutura sagital e $1 \mathrm{~cm}$ anterior à sutura coronal, no hemicrânio direito, por onde foi introduzido o cateter intraparenquimatoso com multissensor (Neurovent-P, Raumedic ${ }^{\circledR}$, Munchberg, Alemanha), que analisa PIC e temperatura cerebral, na substância branca do lobo frontal direito, em uma profundidade de aproximadamente $10 \mathrm{~mm}$. Também foram realizadas trepanação temporal direita posterior e instalação de cateter de monitorização da PIC epidural (Neurodur- $\mathrm{P}^{\circledR}$, Raumedic, Munchberg, Alemanha). Uma trepanação óssea localizada a $1 \mathrm{~cm}$ lateral à sutura sagital e $1 \mathrm{~cm}$ posterior à sutura coronária com diâmetro de $3 \mathrm{~mm}$, a qual permitiu a introdução de uma sonda vesical pediátrica 8 Fr com inclinação de $20^{\circ}$ 
lateral e profundidade de $2 \mathrm{~cm}$, com o objetivo de atingir a substância branca subcortical frontal direita, foi realizada em todos os animais. Controlou-se infusão do balão com a bomba de infusão (B Braun ${ }^{\circledR}$ ) para infusão em 15 minutos. Esse sistema de fluidos foi previamente testado antes da sua instalação definitiva. A solução salina utilizada no experimento foi a solução de $\mathrm{NaCl}$ a 3\%, e o cálculo para infusão é $5,3 \mathrm{~mL} / \mathrm{kg}$ (Figura 1 ).

$\mathrm{O}$ experimento constou de três grupos ( $\mathrm{A}, \mathrm{B}$ e $\mathrm{C})$ com hipertensão intracraniana gerada com a simulação de um hematoma cerebral. Em todos os grupos foram calibrados os parâmetros normais: temperatura do meio ambiente, hemoglobina, $\mathrm{pCO}_{2}$ e saturação de $\mathrm{O}_{2}$. A PIC foi calibrada na instalação do sistema de monitoração. A instalação do sistema de imediato revelava a temperatura cerebral normal e processa-se a calibração da PIC. Esses parâmetros eram avaliados na primeira hora.

\section{Análise estatística}

Os dados sobre PIC intraparenquimatosa, PIC epidural e temperatura cerebral foram obtidos e armazenados em intervalos de um minuto pelo monitor (MPR2 $\log \mathrm{O}$ Raumedic ${ }^{\circledR}$, Munchberg, Alemanha) e, em seguida, transferidos para o software de planilha eletrônica (Statistical Package for Social Sciences), em sua versão 21.0, utilizado para análise estatística.

Para os momentos de aferições comuns a todos os grupos, foram descritas as medidas de PICp e PICe segundo grupos e momentos e comparados os valores das pressões com cada método entre os grupos e momentos com uso de equações de estimação generalizadas com matriz de correlações autorregressiva de ordem 1 entre os momentos. Os resultados foram ilustrados com uso de gráficos de perfis médios com os respectivos erros-padrão segundo grupos e os testes foram realizados com alfa de $5 \%$ e beta de $80 \%$.

\section{Resultados}

Neste estudo utilizamos um modelo de hipertensão intracraniana por balão intracerebral desenvolvido em nossa unidade. Foi realizado um total de 6.480 minutos de monitoração de pressão intracraniana tanto com sistema de aferição de PIC parenquimatosa quanto epidural e somados os registros dos três grupos. Utilizou-se um total de 27 animais, sendo nove em cada grupo de estudo. Os animais utilizados nos experimentos apresentaram peso médio de 19,74 $\pm 1,12 \mathrm{~kg}$, sem diferença de variação entre os grupos. Quatorze animais eram fêmeas e 13, machos. Todos os animais incluídos no estudo completaram os experimentos.

A avaliação por grupo confirmou a eficácia dos modelos de hipertensão intracraniana, com grupo A, gerando condição moderada de elevação da PIC, com a injeção de $4 \mathrm{ml} \mathrm{em} \mathrm{bomba} \mathrm{de} \mathrm{infusão.} \mathrm{Já} \mathrm{no} \mathrm{grupo} B$ também se verificou eficaz elevação da PIC, caracterizando um modelo de reexpansão precoce de hematoma intracraniano (Tabela 1).

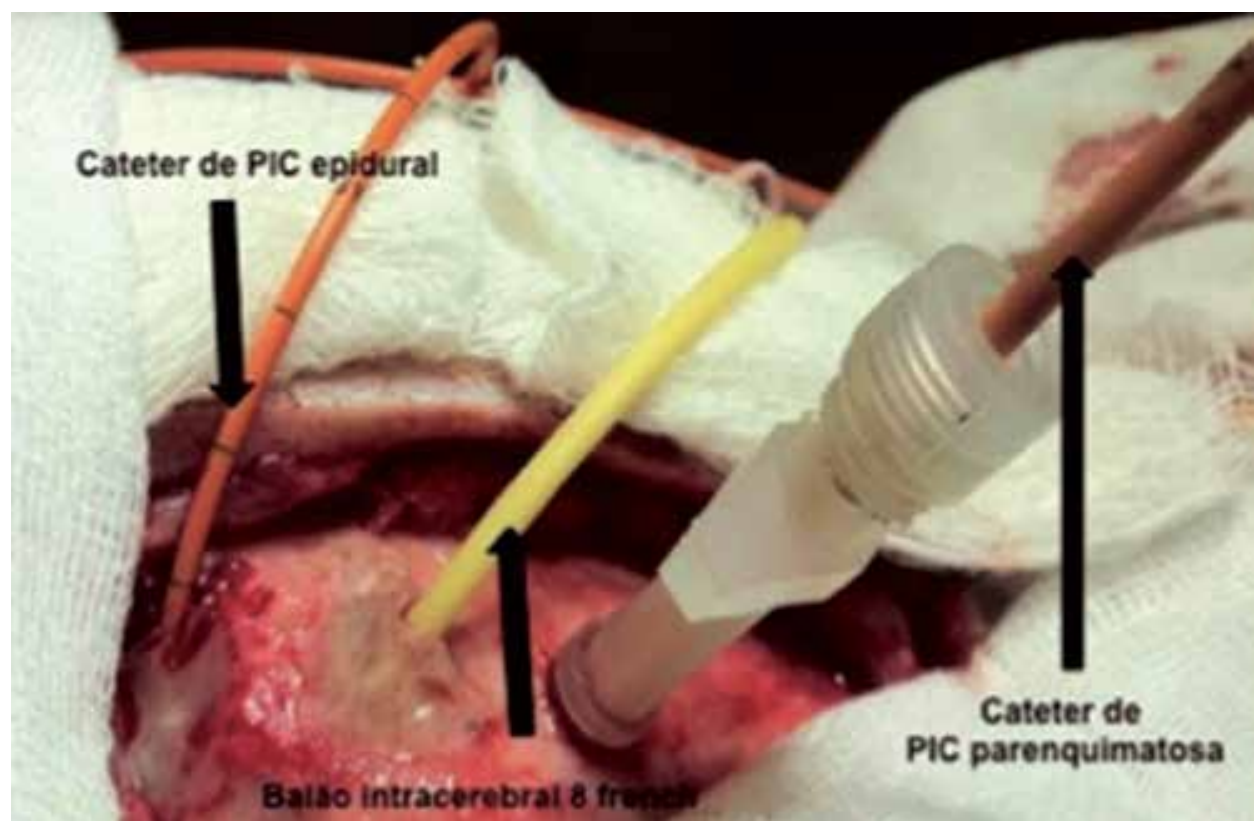

Figura 1 - Imagem do experimento, destacando os catéteres epidural e parenquimatoso e o balão implantado no encéfalo. 
Quanto às variáveis hemodinâmicas, os animais mantiveram-se estáveis durante as fases de avaliação de comparação inicial com marcante elevação da pressão arterial após indução de hipertensão intracraniana nos três grupos, mantendo estabilidade hemodinâmica até a desinsulflação do balão.

Avaliando-se a acurácia comparativa geral, verificou-se adequada correlação intraclasse geral, o que evidencia que os métodos de aferição epidural Neurodur- $\mathrm{P}^{\circledR}$ e parenquimatoso apresentam pressões intracranianas aferidas bastante próximas, uma vez que o coeficiente de correlação intraclasse entre os métodos foi 0,8 (IC95\%: 0745; 0,844) para o total dos dados aferidos (Tabela 2).
Ao analisar a distribuição global dos 27 animais testados com o sistema parenquimatoso e epidural, verificou-se uma tendência a zero, o que evidencia acurácia adequada do sistema epidural utilizando o parenquimatoso como padrão. Contudo, nota-se que, para valores mais altos de pressão intracraniana, os métodos apresentam maior variabilidade do que para valores baixos de pressão, em que os métodos são bastante próximos (Figuras 2 e 3 ).

Analisando as correlações intraclasse após a reprodução de cirurgia, verificou-se perda de acurácia no sistema Neurodur- $\mathrm{P}^{\circledR}$, principalmente no momento de elevada pressão intracraniana, com um coeficiente de correlação intraclasse de 0,559 para o grupo A. Já nos grupos B e

\begin{tabular}{|c|c|c|c|c|c|c|c|c|c|c|}
\hline \multicolumn{11}{|c|}{$\begin{array}{c}\text { Tabela } 1 \text { - Descrição da pressão intracraniana com aferição pelo sistema epidural segundo } \\
\text { grupos e momentos de avaliação e resultado do teste de comparação }\end{array}$} \\
\hline Grupo & Momento & Média & DP & Mediana & Mínimo & Máximo & $\mathbf{N}$ & P grupo & P momento & $P$ interação \\
\hline \multirow{6}{*}{ A } & Basal & 4,2 & 5,0 & 3,8 & 0 & 16 & 9 & & & \\
\hline & Pré-salina & 15,0 & 8,7 & 10,6 & 4 & 27 & 9 & & & \\
\hline & Pós-salina & 11,8 & 8,3 & 6,8 & 4 & 25 & 9 & & & \\
\hline & Pré-cirurgia & 13,3 & 8,4 & 13,5 & 4 & 24 & 9 & & & \\
\hline & Pós-cirurgia & 6,1 & 4,7 & 6,8 & 0 & 12 & 9 & & & \\
\hline & Final & 6,2 & 5,4 & 6,4 & 0 & 17 & 9 & & & \\
\hline \multirow{6}{*}{ B } & Basal & 6,6 & 5,8 & 5 & 0 & 16 & 9 & \multirow{6}{*}{0,067} & \multirow{6}{*}{$<0,001$} & \multirow{6}{*}{0,001} \\
\hline & Pré-salina & 36,1 & 25,5 & 42,2 & 4 & 70 & 9 & & & \\
\hline & Pós-salina & 31,6 & 20,1 & 26,1 & 5 & 63 & 9 & & & \\
\hline & Pré-cirurgia & 31,0 & 18,7 & 29,9 & 5 & 57 & 9 & & & \\
\hline & Pós-cirurgia & 6,7 & 4,2 & 6,7 & 0 & 14 & 9 & & & \\
\hline & Final & 15,0 & 15,4 & 13,7 & 0 & 53 & 9 & & & \\
\hline \multirow{6}{*}{$\mathrm{C}$} & Basal & 8,9 & 5,5 & 8,5 & 4 & 20 & 9 & & & \\
\hline & Pré-salina & 32,0 & 26,0 & 29,4 & 5 & 87 & 9 & & & \\
\hline & Pós-salina & 27,4 & 23,1 & 24,1 & 7 & 84 & 9 & & & \\
\hline & Pré-cirurgia & 31,7 & 28,8 & 23,7 & 5 & 99 & 9 & & & \\
\hline & Pós-cirurgia & 5,5 & 3,7 & 4,6 & 1 & 12 & 9 & & & \\
\hline & Final & 12,2 & 8,6 & 9,4 & 4 & 25 & 9 & & & \\
\hline
\end{tabular}

Tabela 2 - Descrição das medidas de pressão intracraniana em todos os grupos e momentos de avaliação e resultados da medida de concordância

\begin{tabular}{|c|c|c|c|c|c|c|c|c|c|c|}
\hline \multirow[t]{2}{*}{ Grupo/momento } & \multirow[t]{2}{*}{ Variável } & \multirow[t]{2}{*}{ Média } & \multirow[t]{2}{*}{ DP } & \multirow[t]{2}{*}{ P25 } & \multirow[t]{2}{*}{ Mediana } & \multirow[t]{2}{*}{ P75 } & \multirow[t]{2}{*}{$\mathbf{N}$} & \multirow[t]{2}{*}{ CCI } & \multicolumn{2}{|c|}{ IC 95\% } \\
\hline & & & & & & & & & Inferior & Superior \\
\hline Grupo A & PICp & 7,83 & 6,27 & 3,5 & 5,2 & 11,3 & 9 & \multirow{2}{*}{0,559} & \multirow{2}{*}{$-0,079$} & \multirow{2}{*}{0,878} \\
\hline pós-cirurgia & PICe & 6,12 & 4,65 & 0,8 & 6,8 & 10,1 & 9 & & & \\
\hline Grupo B & PICp & 4,97 & 3,85 & 2,4 & 4,8 & 7,9 & 9 & \multirow{2}{*}{0,000} & \multirow{2}{*}{$-0,706$} & \multirow{2}{*}{0,601} \\
\hline pós-cirurgia & PICe & 6,66 & 4,19 & 3,4 & 6,7 & 9,3 & 9 & & & \\
\hline Grupo C & PICp & 5,14 & 5,86 & 0,7 & 2,2 & 10,7 & 9 & \multirow{2}{*}{0,000} & \multirow{2}{*}{$-1,030$} & \multirow{2}{*}{0,336} \\
\hline pós-cirurgia & PICe & 5,47 & 3,69 & 2,9 & 4,6 & 8,5 & 9 & & & \\
\hline \multirow{2}{*}{ Todos } & PICp & 20,97 & 19,27 & 6,7 & 14,6 & 31,6 & 207 & \multirow{2}{*}{0,800} & \multirow{2}{*}{0,745} & \multirow{2}{*}{0,844} \\
\hline & PICe & 19,76 & 20,59 & 5,8 & 12,2 & 25,5 & 207 & & & \\
\hline
\end{tabular}


C, a concordância entre PICp e PICe é baixa nas fases do experimentos com elevada pressão intracraniana.

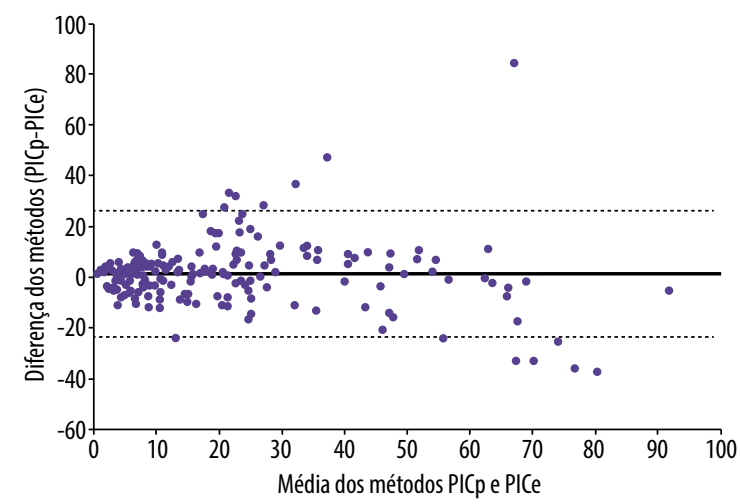

Figura 2 - Distribuição de Bland-Altman das aferições dos métodos epidural e parenquimatoso.

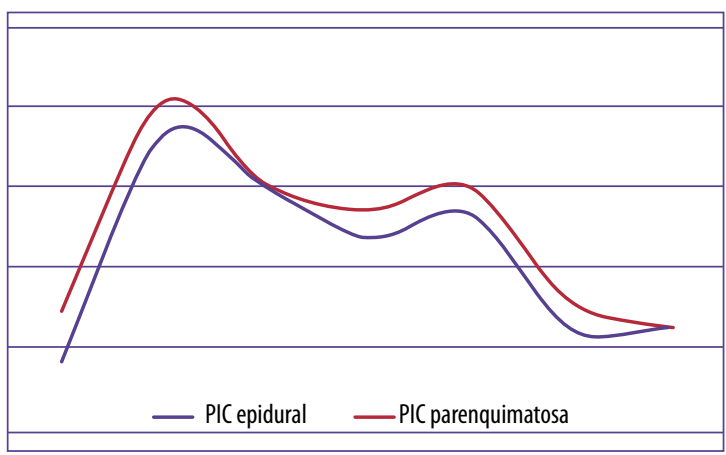

Figura 3 - Distribuição das médias de pressão intracraniana nos animais aferidas pelo sistema de monitoração parenquimatosa e epidural durante os experimentos.

\section{Discussão}

Os resultados deste estudo indicam para uma acurácia moderada de monitoração da PIC por método epidural. Informações mais antigas sobre o uso de monitoração epidural apontavam para erros importantes de aferição que inviabilizaram o uso desse método quando comparado às técnicas de aferição mais invasivas, como ventricular e parenquimatosa. ${ }^{4} \mathrm{O}$ objetivo dos sistemas epidurais de aferição da PIC seria de estabelecer uma técnica precisa e confiável de monitorização quase livre de complicações graves. Na busca desse objetivo, desde a primeira descrição de medição da PIC por sistema epidural por Nornes e Serck-Hanssen ${ }^{4}$, mais de 30 diferentes sensores foram desenvolvidos e testados em estudos experimentais e clínicos. Essa medição epidural não encontrou aceitação universal desde que surgiu, principalmente decorrente de diferenças em relação aos sistemas intraventriculares e parenquimatosos. Apesar do uso de uma variedade de sensores ao longo dos anos, essas diferenças foram descritas por outros autores. ${ }^{5-12}$ Várias hipóteses têm sido propostas para explicar essas observações, tais como o fracasso do transdutor dural com coplanaridade, descolamento dural insuficiente, contato dural incompleto, gradientes de pressão entre os dois locais de medições, fixação insuficiente do transdutor, erros técnicos ou diferenças reais. ${ }^{13-16}$ Contudo, com o melhoramento da tecnologia usada no novo sistema Neurodur- $\mathrm{P}^{\circledR}$, levantaram-se expectativas de um novo modelo que permitisse manter a acurácia dos sistemas parenquimal/ventricular com redução das complicações com uso de transdutor no espaço epidural.

Em nossa avaliação, o transdutor com microssensor strain-gauge apresentou excelente estabilidade no parênquima e espaço peridural. Isso está de acordo com estudos anteriores realizados em diferentes compartimentos. ${ }^{17,18}$ Encontramos uma correlação altamente significativa entre PICs gravadas na amostra geral dos 27 animais nos dois transdutores. Os microssensores epidurais e parenquimatosos apresentaram elevado coeficiente de correlação ( $\mathrm{p}<0,001)$, com uma pequena diferença nas médias em gravações simultâneas no grupo A.

Em comparação aos transdutores de fibra óptica, a principal vantagem dos sensores Neurodur- $\mathrm{P}^{\circledR}$ é sua resistência à manipulação. Poca et al. ${ }^{19}$ ressaltam que os sensores Neurodur- $\mathrm{P}^{\circledR}$ também são confiáveis e fáceis de usar, com uma taxa de complicações baixa. Uma vantagem adicional é que, diferentemente de sensores epidurais mais antigos, os sensores Neurodur- $\mathrm{P}^{\circledR}$ não produziram leituras de PIC artificialmente elevados, tornando os dados mais confiáveis. No grupo $B$, verificamos inclusive valores de médias de leituras consistentes mais baixas nos sistemas epidurais. Raabe et $a .^{20}$, estudando três tipos de sensores epidurais, descreveram falhas com relevância clínica em até um terço dos pacientes, apresentando adequado diagnóstico em avaliação qualitativa. Eide e Sorteberg ${ }^{12}$, ao estudar 12 pacientes com hidrocefalia de pressão intermitente usando o sistema Neurodur- $\mathrm{P}^{\circledR}$, descreveram falhas de aferição clinicamente relevantes em até 33\% dos casos. Em nosso estudo, verificamos perda de acurácia após a indução de hipertensão intracraniana no grupo $\mathrm{C}$ e após a simulação de cirurgia, indicando maior limitação do uso desse sistema no pós-operatório de lesões focais, contudo, no modelo do grupo A e do grupo B, evidenciou-se acurácia adequada para viabilizar o uso clínico em condições de lesões difusas e nas quais os valores de elevação da PIC sejam moderados.

Diante dos resultados, a desvantagem mais importante desse dispositivo é a perda de acurácia nas condições de grandes elevações de PIC, assim como na desinsulflação do balão. Isso pode ser resultado de modificações sobre a dura-máter quando da distensão súbita, assim como algum grau de descolamento dural quando do esvaziamento do balão, gerando uma perda 
de acurácia que pode se manter durante o acompanhamento do animal. Na translação para os pacientes no contexto clínico, poderia determinar um diagnóstico final incorreto do comportamento pós-operatório da PIC.

\section{Conclusão}

O modelo de hipertensão intracraniana por balão em suínos é factível e confiável na geração de hipertensão intracraniana. O sistema de aferição de pressão intracraniana epidural Neurodur- $\mathrm{P}^{\circledR}$ apresenta elevado coeficiente de correlação com o sistema de aferição parenquimatoso no grupo A. Os valores de aferição da pressão intracraniana epidural não apresentam acurácia adequada após a desinsulflação do balão.

\section{Conflitos de interesse}

Os autores declaram não haver conflitos de interesse.

\section{Referências}

1. Smith $M$. Monitoring intracranial pressure in traumatic brain injury. Anesth Analg. 2008;106(1):240-8.

2. Andrade AF, Soares MS, Patriota GC, Belon AR, Paiva WS, Bor-Seng-Shu E, et al. Experimental model of intracranial hypertension with continuous multiparametric monitoring in swine. Arq Neuropsiquiatr. 2013;71(10):802-6.

3. Tanyeri M, Ranka M, Sittipolkul N, Schroeder CM. Microfluidic Wheatstone bridge for rapid sample analysis. Lab Chip. 2011;11(24):4181-6.

4. Nornes H, Serck-Hanssen F. Miniature transducer for intracranial pressure monitoring in man. Acta Neurol Scand. 1970;46(2):203-14.

5. Joergesen PB, Riisheden J. Comparative clinical studies of epidural and ventricular pressure. In: Brock M, Dietz H editors. Intracranial pressure. Berlin: Springer-Verlag; 1972. p. 41-5.

6. Coroneos NJ, McDowall DG, Gibson RM, Pickerodt VW, Keaney NP. Measurement of extradural pressure and its relationship to other intracranial pressures. An experimental and clinical study. J Neurol Neurosurg Psychiatry. 1973;36(4):514-22.

7. Coroneos NJ, McDowall DG, Gibson RM, Pickerodt V, Keaney NP. A comparison of extradural pressure with cerebrospinal fluid pressure. Eur Neurol. 1972;8(1):79-82.
8. Poca MA, Sahuquillo J, Topczewski T, Peñarrubia MJ, Muns A. Is intracranial pressure monitoring in the epidural space reliable? Fact and fiction. J Neurosurg. 2007;106(4):548-56.

9. Pattison K, Wynne-Jones G, Imray CHE. Monitoring intracranial pressure, perfusion and metabolism. Contin Educ Anaesth Crit Care Pain. 2005;5(4):130-3.

10. Powell MP, Crockard HA. Behavior of an extradural pressure monitor in clinical use. Comparison of extradural with intraventricular pressure in patients with acute and chronically raised intracranial pressure. $\mathrm{J}$ Neurosurg. 1985;63(5):745-9.

11. Czosnyka M, Hutchinson PJ, Balestreri M, Hiler M, Smielewski P, Pickard JD. Monitoring and interpretation of intracranial pressure after head injury. Acta Neurochir Suppl. 2006;96:114-8.

12. Eide PK, Sorteberg W. Simultaneous measurements of intracranial pressure parameters in the epidural space and in brain parenchyma in patients with hydrocephalus. $J$ Neurosurg. 2010;113(6):1317-25.

13. Kosteljanetz M, Børgesen SE, Stjernholm P, Christensen L, Osgaard O, Gjerris F, et al. Clinical evaluation of a simple epidural pressure sensor. Acta Neurochir (Wien). 1986;83(34):108-11.

14. Majors R, Schettini A, Mahig J, Nevis AH. Intracranial pressures measured with the coplanar pressure transducer. Med Biol Eng. 1972;10(6):724-33.

15. Pomeranz S, Safar P, Radovsky A, Tisherman SA, Alexander $\mathrm{H}$, Stezoski W. The effect of resuscitative moderate hypothermia following epidural brain compression on cerebral damage in a canine outcome model. J Neurosurg. 1993;79(2):241-51.

16. Schettini A, McKay L, Majors R, Mahig J, Nevis AH. Experimental approach for monitoring surface brain pressure. J Neurosurg. 1971;34(1):38-47.

17. Gopinath SP, Cherian L, Robertson CS, Narayan RK, Grossman RG. Evaluation of a microsensor intracranial pressure transducer. J Neurosci Methods. 1993;49(1-2):11-5.

18. Gopinath SP, Robertson CS, Contant CF, Narayan RK, Grossman RG. Clinical evaluation of a miniature straingauge transducer for monitoring intracranial pressure. Neurosurgery. 1995;36(6):1137-40.

19. Poca MA, Martínez-Ricarte F, Sahuquillo J, Lastra R, Torné $\mathrm{R}$, Armengol MS. Intracranial pressure monitoring with the Neurodur-P epidural sensor: a prospective study in patients with adult hydrocephalus or idiopathic intracranial hypertension. J Neurosurg. 2008;108(5):934-42.

20. Raabe A, Totzauer R, Meyer O, Stöckel R, Hohrein D, Schöche J. Reliability of epidural pressure measurement in clinical practice: behavior of three modern sensors during simultaneous ipsilateral intraventricular or intraparenchymal pressure measurement. Neurosurgery. 1998;43(2):306-11.

Endereço para correspondência

Wellingson Silva Paiva

Rua Alves Guimaraes, 470, ap. 93

05410-000 - São Paulo, SP, Brasil

E-mail: wellingsonpaiva@yahoo.com.br 